\title{
The Structure of Potentially Semi-Stable Deformation Rings
}

\section{Citation}

Kisin, Mark. Forthcoming. The structure of potentially semi-stable deformation rings. Proceedings of the International Congress of Mathematicians, Hyderabad, India, August 19-27, 2010.

\section{Permanent link}

http://nrs.harvard.edu/urn-3:HUL.InstRepos:4794673

\section{Terms of Use}

This article was downloaded from Harvard University's DASH repository, and is made available under the terms and conditions applicable to Open Access Policy Articles, as set forth at http:// nrs.harvard.edu/urn-3:HUL.InstRepos:dash.current.terms-of-use\#OAP

\section{Share Your Story}

The Harvard community has made this article openly available.

Please share how this access benefits you. Submit a story.

Accessibility 


\title{
The structure of potentially semi-stable deformation rings *
}

\author{
Mark Kisin
}

\begin{abstract}
Inside the universal deformation space of a local Galois representation one has the set of deformations which are potentially semi-stable of given $p$-adic Hodge and Galois type. It turns out these points cut out a closed subspace of the deformation space. A deep conjecture due to Breuil-Mézard predicts that part of the structure of this space can be described in terms of the local Langlands correspondence. For 2-dimensional representations the conjecture can be made precise. We explain some of the progress in this case, which reveals that the conjecture is intimately connected to the $p$-adic local Langlands correspondence, as well as to the Fontaine-Mazur conjecture.
\end{abstract}

Mathematics Subject Classification (2000). Primary 00A05; Secondary 00B10.

Keywords.

\section{Introduction}

The study of deformations of Galois representations was initiated by Mazur [Ma]. Already in that article Mazur considered deformations satisfying certain local conditions formulated in terms of $p$-adic Hodge theory. The importance of deformations satisfying such conditions became clear with the formulation of the FontaineMazur conjecture [FM], and the spectacular proof of the Shimura-Taniyama conjecture on modularity of elliptic curves over $\mathbb{Q}$ by Wiles, Taylor-Wiles, and their collaborators [Wi], [TW], [BCDT].

The first question which arises concerns the nature of the subspaces cut out by these conditions: Suppose that $K / \mathbb{Q}_{p}$ is a finite extension with absolute Galois group $G_{K}$, let $\mathbb{F} / \mathbb{F}_{p}$ be a finite extension, and $V_{\mathbb{F}}$ a finite dimensional $\mathbb{F}$-vector space equipped with a continuous, absolutely irreducible $G_{K}$-action. Then $V_{\mathbb{F}}$ admits a universal deformation ring $R_{V_{\mathbb{F}}}$. A closed point $x \in \operatorname{Spec} R_{V_{\mathbb{F}}}[1 / p]$ gives rise to a deformation $L_{x}$ of $V_{\mathbb{F}}$, so that $L_{x} \otimes_{\mathbb{Z}_{p}} \mathbb{Q}_{p}$ is a representation of $G_{K}$ on a finite dimensional vector space over a finite extension of $W(\mathbb{F})[1 / p]$. One can ask whether the points such that $L_{x} \otimes_{\mathbb{Z}_{p}} \mathbb{Q}_{p}$ satisfies the condition are cut out by a closed subspace of $\operatorname{Spec} R_{V_{\mathbb{F}}}[1 / p]$.

\footnotetext{
* It is a pleasure to thank Christophe Breuil, Kevin Buzzard, Toby Gee, James Newton, Vytautas Paskunas, David Savitt and Wansu Kim for useful comments on this paper. The author was partially supported by NSF grant DMS-0701123.
} 
Of course the answer depends on the condition one imposes. In [Fo 2] Fontaine suggests (at least implicitly) that the answer should be affirmative if one requires the representations to become semi-stable over a fixed extension $K^{\prime} / K$ and with Hodge-Tate weights in a fixed interval. Attached to any such representation $V$ is a finite dimensional representation of the inertia subgroup $I_{K} \subset G_{K}$, which, in some sense, measures the failure of $V$ to be semi-stable. One can sharpen Fontaine's conjecture by fixing a representation $\tau$ of $I_{K}$, with open kernel, and requiring $L_{x} \otimes_{\mathbb{Z}_{p}} \mathbb{Q}_{p}$ to have fixed Hodge-Tate weights and associated $I_{K}$-representation $\tau$. That this refined condition cuts out a closed subspace was conjectured in special cases in the papers of Fontaine-Mazur [FM, p191], Breuil-Conrad-DiamondTaylor [BCDT, Conj. 1.1.1], and suggested more generally by Breuil-Mézard [BM, Conj. 1.1, p214].

After partial results by several people (see section 1.2.5 below for a more detailed discussion) such a result was proved in general in [Ki 4]. Thus, for some finite normal extension $\mathcal{O}$ of $W(\mathbb{F})$ one obtains a quotient $R_{V_{\mathbb{F}}}^{\mathbf{v}, \tau}$ of $R_{V_{\mathbb{F}}} \otimes_{W(\mathbb{F})} \mathcal{O}$ whose points in characteristic 0 correspond precisely to deformations of $V_{\mathbb{F}}$ which become semi-stable over some finite extension of $K$, have the chosen fixed Hodge-Tate weights and associated $I_{K}$-representation $\tau .{ }^{1}$

The conjectures of Breuil-Mézard predict a deep connection between the structure of $R_{V_{\mathbb{F}}}^{\mathbf{v}, \tau}$ and the representation theory of $\mathrm{GL}_{d}\left(\mathcal{O}_{K}\right)$, where $d=\operatorname{dim}_{\mathbb{F}} V_{\mathbb{F}}$. ${ }^{2}$ This can be made precise when $V_{\mathbb{F}}$ is two dimensional, which we assume for the rest of this introduction. In this case, a result of Henniart attaches to $\tau$ a smooth, irreducible, finite dimensional representation $\sigma(\tau)$ of $\mathrm{GL}_{2}\left(\mathcal{O}_{K}\right)$ which is characterized in terms of the local Langlands correspondence. On the other hand, the cocharacter $\mathbf{v}$ gives rise to an algebraic representation $\sigma(\mathbf{v})$ of $\mathrm{GL}_{2}\left(\mathcal{O}_{K}\right)$. Let $L_{\mathbf{v}, \tau} \subset \sigma(\mathbf{v}) \otimes \sigma(\tau)$ be a $\mathrm{GL}_{2}\left(\mathcal{O}_{K}\right)$ invariant lattice. Then the conjecture predicts the Hilbert-Samuel multiplicity $e\left(R_{V_{\mathbb{F}}}^{\mathbf{v}, \tau} / \pi\right)$ of $R_{V_{\mathbb{F}}}^{\mathbf{v}, \tau} / \pi$ in terms of the multiplicities of the Jordan-Hölder factors of $L_{\mathbf{v}, \tau} / \pi L_{\mathbf{v}, \tau}$. Here $\pi \in \mathcal{O}$ denotes a uniformizer. Indeed, one can formulate such a conjecture in any dimension assuming an analogue of Henniart's result. When $\tau$ is irreducible a higher dimensional analogue of Henniart's result has been proved by Paskunas $[\mathrm{Pa}]$.

It is slightly more convenient to work with the quotient $R_{V_{\mathbb{F}}}^{\mathbf{v}, \tau, \psi}$ of $R_{V_{\mathbb{F}}}^{\mathbf{v}, \tau}$ which corresponds to deformations having determinant $\psi$ times the cyclotomic character, for some appropriately chosen ${ }^{3} \psi$. The general shape of such a conjecture is then that

$$
e\left(R_{V_{\mathbb{F}}}^{\mathbf{v}, \tau, \psi} / \pi\right)=\sum_{\bar{\sigma}} a(\bar{\sigma}) \mu_{\bar{\sigma}}\left(V_{\mathbb{F}}\right)
$$

where $\bar{\sigma}$ runs over irreducible $\bmod p$ representations of $\mathrm{GL}_{2}(k), k$ the residue field

\footnotetext{
${ }^{1}$ Here the symbol $\mathbf{v}$ indicates a conjugacy class of cocharacters corresponding to the choice of Hodge-Tate weights; we refer to section 1.1.3 below for the precise definition. The choice of $\mathcal{O}$ is related to the field of definition of $\mathbf{v}$ and $\tau$.

${ }^{2}$ Strictly speaking [BM] makes this conjecture in detail for two dimensional representations, $K=\mathbb{Q}_{p}$ and small Hodge-Tate weights. However, the possibility of this connection holding more generally is suggested on p214 of loc. cit.

${ }^{3}$ In order that the quotient is non-zero, one needs a condition of compatibility between $\psi$ and $(\mathbf{v}, \tau)$ (see section 2.2 below) which we assume from now on.
} 
of $K, a(\bar{\sigma})$ denotes the multiplicity of $\bar{\sigma}$ as a Jordan-Hölder factor of $L_{\mathbf{v}, \tau} / \pi L_{\mathbf{v}, \tau}$, and $\mu_{\bar{\sigma}}\left(V_{\mathbb{F}}\right)$ is a non-negative integer. This equality can be viewed as a system of infinitely many equations (corresponding to the choices of $\mathbf{v}$ and $\tau$ ) in the finitely many unknowns $\mu_{\bar{\sigma}}\left(V_{\mathbb{F}}\right)$. One can of course also ask for a version of such a conjecture where the $\mu_{\bar{\sigma}}\left(V_{\mathbb{F}}\right)$ are given explicitly, as is done in $[\mathrm{BM}]$ when $K=\mathbb{Q}_{p}$.

For two dimensional representations and $K=\mathbb{Q}_{p}$ most of the Breuil-Mézard conjecture is proved in $[\mathrm{Ki} 5]$. The proof consists of two parts: One uses the $p$ adic local Langlands correspondence of Breuil and Colmez [Br 1], [Co] to show that $e\left(R_{V_{\mathbb{F}}}^{\mathbf{v}, \tau, \psi} / \pi\right)$ is bounded above by the expected value. A modified form of the Taylor-Wiles patching argument, introduced in [Ki 1$]$, is then used to prove the other inequality. To do this one uses $L_{\mathbf{v}, \tau}$-valued automorphic forms on a totally definite quaternion algebra to construct a module $M_{\infty}$ which is finite of rank $\leq 1$ over a formally smooth $R_{V_{\mathbb{F}}}^{\mathbf{v}, \tau, \psi}$-algebra $R_{\infty}$. Then

$$
e\left(R_{V_{\mathbb{F}}}^{\mathbf{v}, \tau, \psi} / \pi\right)=e\left(R_{\infty} / \pi\right) \geq e\left(M_{\infty} / \pi M_{\infty}\right)
$$

where the final quantity denotes the Hilbert-Samuel multiplicity of the $R_{\infty} / \pi$ module $M_{\infty} / \pi M_{\infty}$. This multiplicity can in turn be analyzed in terms of the Jordan-Hölder factors of $L_{\mathbf{v}, \tau} / \pi L_{\mathbf{v}, \tau}$.

The restriction $K=\mathbb{Q}_{p}$ is used primarily so as to be able to apply the $p$-adic local Langlands correspondence, which is available for $\mathrm{GL}_{2}\left(\mathbb{Q}_{p}\right)$ but remains somewhat elusive for $\mathrm{GL}_{2}(K)$ with $K \neq \mathbb{Q}_{p}$. Indeed the Breuil-Mézard conjecture may be viewed as an avatar of that correspondence. On the other hand, the modified Taylor-Wiles method can be applied without restrictions on $K$. It always gives an inequality involving $e\left(R_{V_{\mathbb{F}}}^{\mathbf{v}, \tau, \psi} / \pi\right)$ with equality being essentially equivalent to a modularity lifting theorem for representations which are of type $(\mathbf{v}, \tau)$ at primes dividing $p$. Such lifting theorems are predicted by the Fontaine-Mazur conjecture and generalize the results used to prove the Shimura-Taniyama conjecture. They were the main motivation of [Ki 5].

In particular, one can try to use modularity lifting theorems to prove cases of the Breuil-Mézard conjecture for $K \neq \mathbb{Q}_{p}$. We give an example of such a result in $\S 3$, using the modularity lifting theorems for potentially Barsotti-Tate representations proved in [Ki 1] and [Ge 1]. The coefficients $\mu_{\bar{\sigma}}\left(V_{\mathbb{F}}\right)$ are not made explicit in this case. One can hope to do that when $K / \mathbb{Q}_{p}$ is unramified, assuming the BuzzardDiamond-Jarvis conjecture $[\mathrm{BDJ}]$ on the weights of automorphic forms giving rise to a given 2-dimensional $\bmod p$ representation. Most of this has been proved by Gee [Ge 2], but one really needs the whole conjecture to determine all the coefficients. Nevertheless, we explain how to use Gee's result to prove the expected lower bound for $e\left(R_{V_{\mathbb{F}}}^{\mathbf{v}, \tau, \psi} / \pi\right)$ when $V_{\mathbb{F}}$ is absolutely irreducible and satisfies a mild additional restriction.

The paper is organized as follows: In $\S 1$ we recall the definition of the rings $R_{V_{\mathbb{F}}}^{\mathbf{v}, \tau, \psi}$ and some of their variants. In $\S 2$, we formulate the general form of the Breuil-Mézard conjecture and recall the explicit definition of $\mu_{\bar{\sigma}}\left(V_{\mathbb{F}}\right)$ when $K / \mathbb{Q}_{p}$ is unramified and $V_{\mathbb{F}}$ is absolute irreducible. In this case these integers are all either 0 or 1 , and the explicit description is essentially a reformulation of the conjecture of [BDJ]. Finally, in $\S 3$ we prove the two theorems on $e\left(R_{V_{\mathbb{F}}}^{\mathbf{v}, \tau, \psi} / \pi\right)$ mentioned above. 


\section{Potentially semi-stable deformation rings}

1.1. Potentially semi-stable representations. Let $K / \mathbb{Q}_{p}$ be a finite extension with residue field $k$, and fix an algebraic closure $\bar{K} / K$. For a subfield $K^{\prime} \subset \bar{K}$, containing $K$, we write $G_{K^{\prime}}=\operatorname{Gal}\left(\bar{K} / K^{\prime}\right)$ and $I_{K^{\prime}} \subset G_{K^{\prime}}$ for the inertia subgroup of $G_{K^{\prime}}$. We denote by $K_{0}^{\prime}$ the maximal absolutely unramified subfield of $K^{\prime}$, and by $\mathcal{O}_{K^{\prime}}$ the ring of integers of $K^{\prime}$.

Recall Fontaine's [Fo 1] period rings

$$
B_{\text {cris }} \subset B_{\text {st }} \subset B_{\mathrm{dR}} \text {. }
$$

The ring $B_{\text {st }}$ is a $\bar{K}_{0}$-algebra, equipped with a Frobenius endomorphism $\varphi$ and an operator $N$ satisfying $N \varphi=p \varphi N$, and we have $B_{\text {cris }}=B_{\mathrm{st}}^{N=0}$. The ring $B_{\mathrm{dR}}$ is a discrete valuation field with residue field $\widehat{\bar{K}}$. In particular, it carries a filtration given by the valuation. The above inclusions induce inclusions

$$
B_{\text {cris }} \otimes_{K_{0}} K \subset B_{\mathrm{st}} \otimes_{K_{0}} K \subset B_{\mathrm{dR}} .
$$

In particular, the rings $B_{\text {cris }} \otimes_{K_{0}} K$ and $B_{\text {st }} \otimes_{K_{0}} K$ are equipped with the filtration induced from $B_{\mathrm{dR}}$.

Suppose that $V$ is a finite dimensional $\mathbb{Q}_{p}$-vector space equipped with a continuous action of $G_{K}$. We set

$$
D_{\text {cris }}(V)=\left(B_{\text {cris }} \otimes_{\mathbb{Q}_{p}} V\right)^{G_{K}}, \quad D_{\text {st }}(V)=\left(B_{\text {st }} \otimes_{\mathbb{Q}_{p}} V\right)^{G_{K}} .
$$

Then $D_{\text {st }}(V)$ is a $K_{0}$-vector space of dimension $\leq \operatorname{dim}_{\mathbb{Q}_{p}} V$ equipped with operators $\varphi$ and $N$, with $\varphi$ a bijection and satisfying $N \varphi=p \varphi N$. We have $D_{\text {cris }}(V)=$ $D_{\text {st }}(V)^{N=0}$. Moreover,

$$
D_{\text {cris }}(V) \otimes_{K_{0}} K \subset D_{\mathrm{st}}(V) \otimes_{K_{0}} K \subset D_{\mathrm{dR}}(V):=\left(B_{\mathrm{dR}} \otimes_{\mathbb{Q}_{p}} V\right)^{G_{K}} .
$$

So $D_{\text {cris }}(V) \otimes_{K_{0}} K$ and $D_{\text {st }}(V) \otimes_{K_{0}} K$ are equipped with a filtration.

A representation $V$ is called crystalline (respectively semi-stable) if $D_{\text {cris }}(V)$ (resp. $\left.D_{\mathrm{st}}(V)\right)$ has $K_{0}$-dimension $\operatorname{dim}_{\mathbb{Q}_{p}} V$, in which case both (resp. the second) inclusions in (1.1.1) are equalities. We say that $V$ is potentially crystalline (resp. potentially semi-stable) if $\left.V\right|_{G_{K^{\prime}}}$ is crystalline (resp. semi-stable) for some finite extension $K^{\prime} / K$.

1.1.2. Fix an algebraic closure $\overline{\mathbb{Q}}_{p}$ of $\mathbb{Q}_{p}$ and let $E \subset \overline{\mathbb{Q}}_{p}$ be a finite extension of $\mathbb{Q}_{p}$ with ring of integers $\mathcal{O}$. Let $V_{E}$ be an $E$-vector space of finite dimension $d$, equipped with a continuous action of $G_{K}$. We assume that $V_{E}$ is potentially semi-stable (viewed as a $\mathbb{Q}_{p}$-representation). Then

$$
D_{\mathrm{pst}}\left(V_{E}\right)=\underline{\lim }_{K^{\prime}}\left(B_{\mathrm{st}} \otimes_{\mathbb{Q}_{p}} V_{E}\right)^{G_{K^{\prime}}}
$$

is a vector space over $\bar{K}_{0}$ of dimension $\operatorname{dim}_{\mathbb{Q}_{p}} V_{E}$. Note that $D_{\text {pst }}\left(V_{E}\right)$ is a $\bar{K}_{0} \otimes_{\mathbb{Q}_{p}} E$ module equipped with a semi-linear action of $G_{K}$, and so with a linear action of $I_{K}$. Since $\varphi$ is a bijection on $D_{\text {pst }}\left(V_{E}\right)$, this is necessarily a free $\bar{K}_{0} \otimes_{\mathbb{Q}_{p}} E$-module, 
and since the action of $\varphi$ commutes with that of $I_{K}$, we have $\operatorname{tr}\left(\sigma \mid D_{\text {pst }}\left(V_{E}\right)\right) \in E$ for any $\sigma \in I_{K}$.

Let $\tau: I_{K} \rightarrow \mathrm{GL}_{d}\left(\overline{\mathbb{Q}}_{p}\right)$ be a representation with open kernel. We say that $V_{E}$ is of Galois type $\tau$ if the $I_{K}$-representation $D_{\text {pst }}\left(V_{E}\right)$ is equivalent to $\tau$. That is, $\overline{\mathbb{Q}}_{p} \otimes_{E} D_{\text {pst }}\left(V_{E}\right)$, equipped with its $I_{K}$ action is isomorphic to $\tau \otimes_{\mathbb{Q}_{p}} \bar{K}_{0}$. Concretely this means that for any $\sigma \in I_{K}, \operatorname{tr}\left(\sigma \mid D_{\text {pst }}\left(V_{E}\right)\right)=\operatorname{tr}(\tau(\sigma))$.

We can extend this definition to finite local $E$-algebras $B$ : If $V_{B}$ is a finite free $B$-module, equipped with a continuous, potentially semi-stable action of $G_{K}$, then $D_{\text {pst }}\left(V_{B}\right)$ gives rise to a representation of $I_{K}$ on a finite free $\bar{K}_{0} \otimes_{\mathbb{Q}_{p}} B$-module with traces in $B$. We say that $V_{B}$ is of Galois type $\tau$ if the traces of elements of $I_{K}$ acting on $D_{\text {pst }}\left(V_{B}\right)$ and $\tau$ are equal. If $B$ has residue field $E$ then a potentially semi-stable $V_{B}$ is of type $\tau$ if and only if $V_{B} \otimes_{B} E$ is.

1.1.3. Let $\mathbf{v}$ be a conjugacy class of cocharacters of $\operatorname{Res}_{K / \mathbb{Q}_{p}} \mathrm{GL}_{d}$ (defined over $\left.\overline{\mathbb{Q}}_{p}\right)$. Concretely, v consists of the data of a $d$-tuple of integers for each embedding $K \hookrightarrow \overline{\mathbb{Q}}_{p}$. Let $E_{\mathbf{v}} \subset \bar{E}$ denote the reflex field of $\mathbf{v}$. That is, $E_{\mathbf{v}}$ is the fixed field of the group of $\sigma \in \operatorname{Gal}\left(\overline{\mathbb{Q}}_{p} / \mathbb{Q}_{p}\right)$ such that $\sigma^{*}(\mathbf{v})=\mathbf{v}$. Then $\mathbf{v}$ has a representative defined over $E_{\mathbf{v}}$.

Now let $V_{E}$ be as above, and suppose that $E \supset E_{\mathbf{v}}$. We say that $V_{E}$ has $p$-adic Hodge type $\mathbf{v}$, if the filtration on the $K \otimes_{\mathbb{Q}_{p}} E$-module $D_{\mathrm{dR}}\left(V_{E}\right)$ is induced by the inverse of a cocharacter in the conjugacy class $\mathbf{v}$. As in section 1.1.2, we can extend this definition to representations of $G_{K}$ on finite local $E$-algebras $B$.

1.1.4. Suppose that $V_{E}$ is of $p$-adic Hodge type $\mathbf{v}$, and Galois type $\tau$. An extension of $V_{E}$ by $V_{E}$ in the category of $G_{K}$-representations can be regarded as a representation of $G_{K}$ on a finite free module $V_{E[\epsilon]}$ over the dual numbers $E[\epsilon]$. If $V_{E[\epsilon]}$ is potentially semi-stable it is necessarily of $p$-adic Hodge type $\mathbf{v}$ and Galois type $\tau$. We can compute the space of such extensions as follows: First observe that

$$
\operatorname{ad} D_{\mathrm{pst}}\left(V_{E}\right) \stackrel{\sim}{\longrightarrow} D_{\mathrm{pst}}\left(\operatorname{ad} V_{E}\right) \subset D_{\mathrm{dR}}\left(\operatorname{ad} V_{E}\right) \otimes_{K} \bar{K} \stackrel{\sim}{\longrightarrow} \operatorname{ad} D_{\mathrm{dR}}\left(V_{E}\right) \otimes_{K} \bar{K}
$$

where ad denotes the adjoint so that, for example, $\operatorname{ad} V_{E}=\operatorname{Hom}_{E}\left(V_{E}, V_{E}\right)$. Hence

$$
\left(\operatorname{ad} D_{\mathrm{pst}}\left(V_{E}\right)\right)^{G_{K}} \subset \operatorname{ad} D_{\mathrm{dR}}\left(V_{E}\right) .
$$

Suppose for a moment that $V_{E}$ is potentially crystalline. Then it turns out that the space $\operatorname{Ext}_{\text {pcris }}^{1}\left(V_{E}, V_{E}\right)$ of self extensions of $V_{E}$ which are potentially crystalline is canonically isomorphic to the $H^{1}$ of the following complex concentrated in degrees 0 and 1

$$
\left(\operatorname{ad} D_{\mathrm{pst}}\left(V_{E}\right)\right)^{G_{K}} \stackrel{(1-\varphi, \text { can })}{\longrightarrow}\left(\operatorname{ad} D_{\mathrm{pst}}\left(V_{E}\right)\right)^{G_{K}} \oplus \operatorname{ad} D_{\mathrm{dR}}\left(V_{E}\right) / \operatorname{Fil}^{0} \operatorname{ad} D_{\mathrm{dR}}\left(V_{E}\right),
$$

where the second component of the map is induced by the inclusion (1.1.5). The kernel of this map is canonically isomorphic to $\left(\operatorname{ad} V_{E}\right)^{G_{K}}$. In particular, we have

$$
\operatorname{dim}_{E} \operatorname{Ext}_{\mathrm{pcris}}^{1}\left(V_{E}, V_{E}\right)=\operatorname{dim}_{E} \operatorname{ad} D_{\mathrm{dR}}\left(V_{E}\right) / \operatorname{Fil}^{0} \operatorname{ad} D_{\mathrm{dR}}\left(V_{E}\right)+\operatorname{dim}_{E}\left(\operatorname{ad} V_{E}\right)^{G_{K}}
$$


In particular, if $V_{E}$ is absolutely irreducible, then the right hand side of (1.1.6) depends only on the $p$-adic Hodge type, and is equal to $1+w_{\mathbf{v}}^{>0}$, where $w_{\mathbf{v}}^{>0}$ is the dimension of the Lie subalgebra of $\operatorname{Res}_{K / \mathbb{Q}_{p}} \mathfrak{g} l_{d}$ on which a fixed representative of $\mathbf{v}$ acts with positive weights.

Now suppose that $V_{E}$ is potentially semi-stable. Then the space $\operatorname{Ext}_{\text {pst }}^{1}\left(V_{E}, V_{E}\right)$ of potentially semi-stable self extensions is canonically isomorphic to $H^{1}$ of the total complex (concentrated in degrees $0,1,2$ ) of

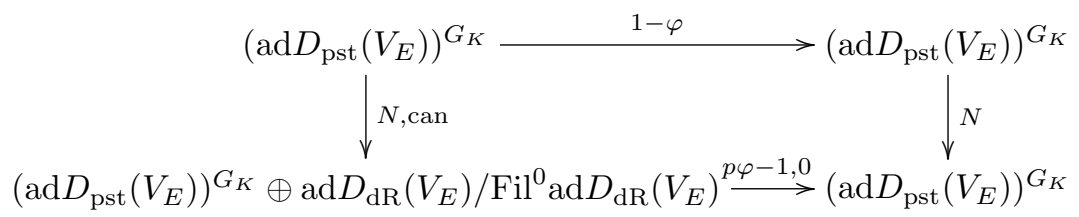

If $V_{E}$ is absolutely irreducible, we deduce that the dimension of $\operatorname{Ext}_{\text {pst }}^{1}\left(V_{E}, V_{E}\right)$ is again $1+w_{\mathbf{v}}^{>0}$ provided the $H^{2}$ of the above total complex vanishes. In general, this $H^{2}$ contains obstructions for the deformation theory of $V_{E}$ as a potentially semi-stable representation.

1.2. Deformation rings. Now let $\overline{\mathbb{F}}_{p}$ be the residue field of $\overline{\mathbb{Q}}_{p}$, and $\mathbb{F} \subset \overline{\mathbb{F}}_{p}$ a finite extension of $\mathbb{F}_{p}$. Let $V_{\mathbb{F}}$ be an $\mathbb{F}$-vector space of dimension $d$ equipped with a continuous action of $G_{K}$. Let $\mathfrak{A} \mathfrak{R}_{W(\mathbb{F})}$ denote the category of Artinian $W(\mathbb{F})$ algebras with residue field $\mathbb{F}$. If $A$ is in $\mathfrak{A} \mathfrak{R}_{W(\mathbb{F})}$, a deformation of $V_{\mathbb{F}}$ to $A$ is a finite free $A$-module equipped with a continuous action of $G_{K}$ and a $G_{K}$-equivariant isomorphism $V_{A} \otimes_{A} \mathbb{F} \stackrel{\sim}{\longrightarrow} V_{\mathbb{F}}$. We denote by $D_{V_{\mathbb{F}}}(A)$ the set of isomorphism classes of deformations of $V_{\mathbb{F}}$ to $A$.

If we fix a basis for $V_{\mathbb{F}}$, then a framed deformation is a deformation $V_{A}$ of $V_{\mathbb{F}}$ to $A$, together with a lifting to $V_{A}$ of the chosen basis of $V_{\mathbb{F}}$. We denote by $D_{V_{\mathbb{F}}}^{\square}(A)$ the set of isomorphism classes of framed deformations of $V_{\mathbb{F}}$ to $A$.

The functor $D_{V_{\mathbb{F}}}^{\square}$ is always pro-representable by a complete local $W(\mathbb{F})$-algebra $R_{V_{\mathbb{F}}}^{\square}$. If End $\operatorname{En}_{\mathbb{F}\left[G_{K}\right]} V_{\mathbb{F}}=\mathbb{F}$ then the functor $D_{V_{\mathbb{F}}}$ is pro-representable by a complete local $W(\mathbb{F})$-algebra $R_{V_{\mathbb{F}}}[\mathrm{Ma}]$. In this case the canonical morphism $R_{V_{\mathbb{F}}} \rightarrow R_{V_{\mathbb{F}}}^{\square}$ is formally smooth.

Now let $E \subset \overline{\mathbb{Q}}_{p}$ be a finite extension of $\mathbb{Q}_{p}$ as before, and assume that the residue field of $E$ contains $\mathbb{F}$. Fix a representation $\tau: I_{K} \rightarrow \mathrm{GL}_{d}(E)$ with open kernel, and a $p$-adic Hodge type $\mathbf{v}$ such that $E_{\mathbf{v}} \subset E$. The main result of $[\mathrm{Ki}$ 4] is that $R_{V_{\mathbb{F}}}^{\square}$ and $R_{V_{\mathbb{F}}}$ (when it is defined) admit quotients which parameterize potentially semi-stable deformations of $V_{\mathbb{F}}$ of Galois type $\tau$ and $p$-adic Hodge type v.

Theorem 1.2.1. There exists a p-torsion free quotient $R_{V_{\mathbb{F}}}^{\square, \tau, \mathbf{v}}$ of $R_{V_{\mathbb{F}}}^{\square} \otimes_{W(\mathbb{F})} \mathcal{O}$ such that for any finite local E-algebra $B$, and any homomorphism $\xi: R_{V_{\mathbb{F}}}^{\square} \rightarrow B$, the $B$-representation of $G_{K}$ induced by $\xi$ is potentially semi-stable of Galois type $\tau$ and p-adic Hodge type $\mathbf{v}$ if and only if $\xi$ factors through $R_{V_{\mathbb{F}}}^{\square, \tau, \mathbf{v}}$.

The irreducible components of $\operatorname{Spec} R_{V_{\mathbb{F}}}^{\square, \tau, \mathbf{v}}[1 / p]$ are generically reduced and of dimension $d^{2}+w_{\mathbf{v}}^{>0}$. 
If $\operatorname{End}_{\mathbb{F}\left[G_{K}\right]} V_{\mathbb{F}}=\mathbb{F}$, then there exists an analogous quotient $R_{V_{\mathbb{F}}}^{\tau, \mathbf{v}}$ of $R_{V_{\mathbb{F}}}$, except that the components of $\operatorname{Spec} R_{V_{\mathbb{F}}^{\tau}}^{\tau, \mathbf{v}}[1 / p]$ have dimension $1+w_{\mathbf{v}}^{>0}$.

We have a completely analogous statement for potentially crystalline representations, except that one can then make a more precise statement about the local structure of the generic fibres of the corresponding rings:

Theorem 1.2.2. There exists a p-torsion free quotient $R_{V_{\mathbb{F}}, \mathrm{cr}}^{\square, \tau, \mathbf{v}}$ of $R_{V_{\mathbb{F}}}^{\square} \otimes_{W(\mathbb{F})} \mathcal{O}$ such that for any finite local E-algebra $B$, and any homomorphism $\xi: R_{V_{\mathbb{F}}}^{\square} \rightarrow B$, the $B$-representation of $G_{K}$ induced by $\xi$ is potentially crystalline of Galois type $\tau$ and p-adic Hodge type $\mathbf{v}$ if and only if $\xi$ factors through $R_{V_{\mathbb{F}}, \mathrm{cr}}^{\square}$.

The irreducible components of Spec $R_{V_{\mathbb{F}}, \mathrm{cr}}^{\square, \tau, \mathbf{v}}[1 / p]$ are formally smooth of dimension $d^{2}+w_{\mathbf{v}}^{>0}$.

If $\operatorname{End}_{\mathbb{F}\left[G_{K}\right]} V_{\mathbb{F}}=\mathbb{F}$, then there exists an analogous quotient $R_{V_{\mathbb{F}}, \mathrm{cr}}^{\tau, \mathbf{v}}$ of $R_{V_{\mathbb{F}}}$, except that the components of $\operatorname{Spec} R_{V_{\mathbb{F}}, \mathrm{cr}}^{\tau, \mathbf{v}}[1 / p]$ have dimension $1+w_{\mathbf{v}}^{>0}$.

Note that it is clear that, if the above quotients exist, then they are unique. The reason for taking $B$ a finite local $E$-algebra, rather than just a finite field extension of $E$, was to ensure this uniqueness.

1.2.3. For $\tau$ trivial, the above results were previously known in special cases: In each of those cases what was actually shown were special cases of the following conjecture of Fontaine [Fo 2]:

Conjecture 1.2.4. (Fontaine) Let $a \leq b$ be integers and $V$ a continuous representation of $G_{K}$ on a finite free $\mathbb{Z}_{p}$-module. Suppose that for $n \geq 1 \mathrm{~V} / \mathrm{p}^{n} \mathrm{~V}$ is a subquotient of a $G_{K}$-stable lattice in a semi-stable (resp. crystalline) representation $V_{n}$ whose Hodge-Tate weights are in $[a, b]$. Then $V \otimes_{\mathbb{Z}_{p}} \mathbb{Q}_{p}$ is semi-stable (resp. crystalline) with Hodge-Tate weights in $[a, b]$.

1.2.5. For crystalline deformations this was shown by Ramakrishna [Ra] when $[a, b]=[0,1]$, using results of Raynaud, ${ }^{4}$ by Fontaine-Lafaille [FL] when $K=K_{0}$ and $[a, b]=[0, p-2]$, and by Berger [Be] whenever $K=K_{0}$. For semi-stable representations with $\left[K: K_{0}\right]|b-a|<p-1$ this is a result of Breuil [Br 2].

The results of [Ki 4], are not proved via Fontaine's conjecture. Rather the quotients $R_{V_{\mathbb{F}}}^{\square, \mathbf{v}, \tau}$ are constructed more directly using the results of [Ki 2] on Galois stable lattices in semi-stable representations. On the other hand, T. Liu has also used the theory of [Ki 2] to prove Fontaine's conjecture in general [Li].

\section{The Breuil-Mézard conjecture}

2.1. Local Langlands and $\boldsymbol{I}_{\boldsymbol{K}}$-representations. From now on we fix a normalization of local class field theory so that the restriction of the cyclotomic

\footnotetext{
${ }^{4}$ Actually, what Ramakrishna shows is that if $V_{n}$ arises from a $p$-divisible group then so does $V$. It was a later result of Breuil that $V$ arises from a $p$-divisible group if and only if it is crystalline with Hodge-Tate weights in $[0,1]$.
} 
character $\chi_{\text {cyc }}: G_{K} \rightarrow \mathbb{Z}_{p}$ to $\mathcal{O}_{K}^{\times} \subset G_{K}$ is given by the norm $N_{K / \mathbb{Q}_{p}}$. This corresponds to the normalization of global class field theory which takes uniformizers to geometric Frobenii.

Consider a representation $\tau: I_{K} \rightarrow \mathrm{GL}_{2}\left(\overline{\mathbb{Q}}_{p}\right)$ with open kernel as in section 1.1.2 We will assume that $\tau$ is the restriction to $I_{K}$ of a 2-dimensional representation of the Weil-Deligne group $\mathrm{WD}_{K}$ of $K$.

If $\tilde{\tau}$ is any continuous, Frobenius semi-simple 2-dimensional representation of $\mathrm{WD}_{K}$, we denote by $\pi(\tilde{\tau})$ the representation of $\mathrm{GL}_{2}(K)$ attached to $\tilde{\tau}$ by the local Langlands correspondence ${ }^{5}$, normalized so that $\pi(\tilde{\tau})$ has central character $\left.\operatorname{det} \tilde{\tau}\right|_{K \times}|\cdot|^{-1}$. We have the following result [BM, Appendix].

Theorem 2.1.1. (Bushnell-Kutzko, Henniart) There is a finite dimensional, irreducible $\overline{\mathbb{Q}}_{p}$-representation $\sigma(\tau)$ (resp. $\quad \sigma_{\mathrm{cr}}(\tau)$ ) of $\mathrm{GL}_{2}\left(\mathcal{O}_{K}\right)$ such that for any 2-dimensional, Frobenius semi-simple representation $\tilde{\tau}$ of $\mathrm{WD}_{K},\left.\pi(\tilde{\tau})\right|_{\mathrm{GL}_{2}\left(\mathcal{O}_{K}\right)}$ contains $\sigma(\tau)$ (resp. $\sigma_{\mathrm{cr}}(\tau)$ ) if and only if $\left.\tilde{\tau}\right|_{I_{K}} \sim \tau$ (resp. $\left.\tilde{\tau}\right|_{I_{K}} \sim \tau$ and $N=0$ on $\tilde{\tau})$.

The representation $\sigma(\tau)$ (resp. $\sigma_{\mathrm{cr}}(\tau)$ ) is uniquely determined by this property except possibly ${ }^{6}$ when $|k|=2$.

2.1.2. Let $\mathbf{v}$ be a cocharacter of $\operatorname{Res}_{K / \mathbb{Q}_{p}} \mathrm{GL}_{2}$ and suppose that $E$ contains the image of all embeddings $K \hookrightarrow \overline{\mathbb{Q}}_{p}$. In particular, $E_{\mathbf{v}} \subset E$. Concretely, v consists of the data of a pair of integers $\left(w_{\iota}, k_{\iota}+w_{\iota}\right)$ with $k_{\iota} \geq 0$, for each embedding $\iota: K \hookrightarrow \overline{\mathbb{Q}}_{p}$. We say that $\mathbf{v}$ is regular if $k_{\iota} \geq 1$ for all $\iota$. For a regular $\mathbf{v}$ we set

$$
\sigma(\mathbf{v})=\otimes_{\iota: K \hookrightarrow E} \iota^{*}\left(\operatorname{Sym}^{k_{\iota}-1} K^{2} \otimes \operatorname{det}^{w_{\iota}}\right)
$$

Now suppose that $\tau, \sigma(\tau)$ and $\sigma_{\mathrm{cr}}(\tau)$ are defined over $E$. We again denote by $\sigma(\tau)$ and $\sigma_{\mathrm{cr}}(\tau)$ the corresponding $E$-vector spaces. Then we set $\sigma(\mathbf{v}, \tau)=$ $\sigma(\tau) \otimes_{E} \sigma(\mathbf{v})$, and $\sigma_{\mathrm{cr}}(\mathbf{v}, \tau)=\sigma_{\mathrm{cr}}(\tau) \otimes_{E} \sigma(\mathbf{v})$.

2.2. Formulation of the conjecture. Let $\varpi$ be a uniformizer of $K$, and $\chi_{\varpi}$ the Lubin-Tate character attached to $\varpi$. For $\mathbf{v}$ as above we set

$$
\chi_{\mathbf{v}}=\prod_{\iota: K \hookrightarrow E}\left(\iota \circ \chi_{\varpi}\right)^{k_{\iota}+2 w_{\iota}-1} .
$$

Now fix $\tau$ as in section 2.1 and $\mathbf{v}$ as above. Let $\psi: G_{K} \rightarrow \mathcal{O}^{\times}$be a continuous character such that $\left.\psi\right|_{I_{K}}=\left.\chi_{\mathbf{v}}\right|_{I_{K}} \cdot \operatorname{det} \tau$.

Let $\mathbb{F} \subset \overline{\mathbb{F}}_{p}$ be the residue field of $E$, and let $V_{\mathbb{F}}$ be a two dimensional $\mathbb{F}$-vector space equipped with a continuous action of $G_{K}$ such that the determinant of $V_{\mathbb{F}}$ is equal to the reduction of $\psi \chi_{\text {cyc. }}$.

\footnotetext{
${ }^{5}$ If $\tilde{\tau} \sim \chi \oplus \chi|\cdot|$ for some character $\chi$ of $\mathrm{WD}_{K}$, then we take $\pi(\tilde{\tau})$ to be the reducible principal series representation $\chi \circ \operatorname{det} \otimes \operatorname{Ind}_{B}^{\mathrm{GL}_{2}(K)} \mathbf{1}$ where $B \subset \mathrm{GL}_{2}(K)$ is a Borel, rather than the more classical choice of the one dimensional representation $\chi \circ$ det .

${ }^{6}$ More precisely, if $|k|=2$ and $\tau \sim \chi \oplus \chi \varepsilon_{0}$ with $\varepsilon_{0}$ a ramified character then there are two such representations. In this case, we take $\sigma(\tau)=\sigma_{\mathrm{cr}}(\tau)$ to be $\chi \circ$ det times the representation denoted by $u_{N_{0}}\left(\varepsilon_{0}\right)$ in [He, A.2.2]. A more adventurous conjecture below would be to allow $\sigma(\tau)$ and $\sigma_{\mathrm{cr}}(\tau)$ to be either of the two representations having the property in the theorem.
} 
We denote by $R_{V_{\mathbb{F}}}^{\square, \mathbf{v}, \tau, \psi}$ the quotient of the ring $R_{V_{\mathbb{F}}}^{\square, \mathbf{v}, \tau}$ introduced in Theorem 1.2.1 corresponding to deformations with determinant (the image of) $\psi \chi_{\mathrm{cyc}}$. Similarly we have the ring $R_{V_{\mathbb{F}}, \mathrm{cr}}^{\square, \mathbf{v}, \psi}$ and, when $\operatorname{End}_{\mathbb{F}\left[G_{K}\right]} V_{\mathbb{F}}=\mathbb{F}$, the rings $R_{V_{\mathbb{F}}}^{\mathbf{v}, \tau, \psi}$ and $R_{V_{\mathrm{F}}, \mathrm{cr}}^{\mathrm{v}, \tau, \psi}$

Let $\pi \subset \mathcal{O}$ be a uniformizer. We want to relate the Hilbert-Samuel multiplicity of the ring $R_{V_{\mathbb{F}}}^{\square, v, \tau, \psi} / \pi$ and its variants to the reduction mod $\pi$ of a $\operatorname{GL}_{2}\left(\mathcal{O}_{K}\right)$ stable $\mathcal{O}$-lattice $L_{\mathbf{v}, \tau} \subset \sigma(\mathbf{v}, \tau)$. To do this we need to recall the irreducible $\bmod p$ representations of $\mathrm{GL}_{2}(k)[\mathrm{BL}]$.

2.2.1. Let $\underline{n}=\left\{n_{\bar{\iota}}\right\}$ and $\underline{m}=\left\{m_{\bar{\iota}}\right\}$ be tuples of integers indexed by the embeddings $\bar{\iota}: k \hookrightarrow \mathbb{F}$, with $0 \leq n_{\bar{\iota}}, m_{\bar{\iota}} \leq p-1$ and not all $m_{\bar{\iota}}=p-1$. Then the representations

$$
\sigma_{\underline{n}, \underline{m}}=\otimes_{\bar{\iota}} \bar{\iota}^{*}\left(\operatorname{Sym}^{n_{\bar{\iota}}} k^{2} \otimes \operatorname{det}^{m_{\bar{\iota}}}\right)
$$

are irreducible and pairwise distinct, and any irreducible $\bmod p$ representation of $\mathrm{GL}_{2}(k)$ is isomorphic to one of the $\sigma_{\underline{n}, \underline{m}}$. These are also the irreducible mod $p$ representations of $\mathrm{GL}_{2}\left(\mathcal{O}_{K}\right)$.

2.2.2. Recall that the Hilbert-Samuel multiplicity is an invariant which measures the complexity of a Noetherian, local ring $A$. If $A$ has dimension $d$ and maximal ideal $\mathfrak{m} \subset A$ then, for sufficiently large $n$, the function $n \mapsto \ell\left(A / \mathfrak{m}^{n+1}\right)$ is a polynomial of degree $d$, where $\ell$ denotes length. Then the Hilbert-Samuel multiplicity $e(A)$ is defined as $d$ ! times the coefficient of $X^{d}$ in this polynomial. It is necessarily an integer.

More generally, if $M$ is a finite $A$-module, then for $n$ sufficiently large, $n \mapsto$ $\ell\left(M / \mathfrak{m}^{n+1}\right)$ is a polynomial of degree at most $d$. The coefficient of $X^{d}$ has the form $e_{A}(M) / d$ ! for a non-negative integer $e_{A}(M)$ which is called the Hilbert-Samuel multiplicity of $M$.

The following is a natural generalization of the Breuil-Mézard conjecture which is, to some extent, already hinted at in [BM, p214].

Conjecture 2.2.3. There exist integers $\mu_{\underline{n}, \underline{m}}\left(V_{\mathbb{F}}\right)$ such that for any $\tau$ and $\mathbf{v}$, and $\psi$ as above, with $\mathbf{v}$ regular, we have

$$
e\left(R_{V_{\mathbb{F}}}^{\square, \mathbf{v}, \tau, \psi} / \pi\right)=\sum_{\underline{n}, \underline{m}} a(\underline{n}, \underline{m}) \mu_{\underline{n}, \underline{m}}\left(V_{\mathbb{F}}\right),
$$

where

$$
\left(L_{\mathbf{v}, \tau} \otimes_{\mathcal{O}} \mathbb{F}\right)^{\mathrm{sS}} \stackrel{\sim}{\longrightarrow} \oplus_{\underline{n}, \underline{\underline{m}}} \sigma_{\underline{n}, \underline{\underline{n}}^{a(\underline{\underline{n}})}} .
$$

Similarly, if $L_{\mathbf{v}, \tau}^{\mathrm{cr}}$ is a $\mathrm{GL}_{2}\left(\mathcal{O}_{K}\right)$-stable lattice in $\sigma_{\mathrm{cr}}(\mathbf{v}, \tau)$ then

$$
e\left(R_{V_{\mathbb{F}}, \mathrm{v}, \tau, \psi}^{\square} / \pi\right)=\sum_{\underline{n}, \underline{m}} a(\underline{n}, \underline{m}) \mu_{\underline{n}, \underline{m}}\left(V_{\mathbb{F}}\right),
$$

where

$$
\left(L_{\mathbf{v}, \tau}^{\mathrm{cr}} \otimes_{\mathcal{O}} \mathbb{F}\right)^{\mathrm{ss}} \stackrel{\sim}{\longrightarrow} \oplus_{\underline{n}, \underline{\underline{m}}} \sigma_{\underline{n}, \underline{\underline{n}}}^{a(\underline{\underline{m}})} .
$$


2.2.4. Note that when $V_{\mathbb{F}}$ has trivial endomorphisms, the morphism $R_{V_{\mathbb{F}}}^{\mathbf{v}, \tau, \psi} \rightarrow$ $R_{V_{\mathbb{F}}}^{\square, \mathbf{v}, \tau, \psi}$ (resp. $R_{V_{\mathbb{F}}, \mathrm{cr}}^{\mathbf{v}, \tau, \psi} \rightarrow R_{V_{\mathbb{F}}, \mathbf{v}, \tau, \psi}^{\square}$ ) is formally smooth, so the Hilbert-Samuel multiplicities of these two rings are equal.

The equalities in Conjecture 2.2.3 can be viewed as an infinite number of equations (corresponding to the choices of $\mathbf{v}$ and $\tau$ ) in the finitely many unknowns $\mu_{\underline{n}, \underline{m}}\left(V_{\mathbb{F}}\right)$. If these equalities hold, then the $\mu_{\underline{n}, \underline{m}}\left(V_{\mathbb{F}}\right)$ may be determined by taking $\tau$ trivial, and selecting $\mathbf{v}$ as follows: Choose a subset $L$ of the set of embeddings $K \hookrightarrow E$ such that $L$ maps bijectively onto the set of embeddings $k \hookrightarrow \mathbb{F}$. Define $\mathbf{v}$ by $k_{\iota}=n_{\bar{\iota}}+1$ and $w_{\iota}=m_{\iota}$ if $\iota \in L$ and $k_{\iota}=1, w_{\iota}=0$ otherwise. Here $\bar{\iota}$ denotes the reduction of $\iota$. Then $\sigma_{\mathrm{cr}}(\tau)$ is the trivial representation of $\mathrm{GL}_{2}\left(\mathcal{O}_{K}\right)$ and any $\mathrm{GL}_{2}\left(\mathcal{O}_{K}\right)$-stable lattice $L_{\mathbf{v}, \tau}^{\mathrm{cr}}$ in $\sigma_{\mathrm{cr}}(\mathbf{v}, \tau)$, has reduction isomorphic to $\sigma_{\underline{n}, \underline{m}}$. So Conjecture 2.2 .3 predicts

$$
\mu_{\underline{n}, \underline{m}}\left(V_{\mathbb{F}}\right)=e\left(R_{\mathrm{cr}}^{\square, \mathbf{v}, \tau, \psi} / \pi\right) .
$$

2.3. The case of an unramified extension. When $K / \mathbb{Q}_{p}$ is unramified, the integers on the right hand side of (2.2.5) can be determined in almost all cases, and are usually in $\{0,1,2\}$. In this case, the condition that $\mu_{\underline{n}, \underline{m}}\left(V_{\mathbb{F}}\right) \neq 0$ is closely related to the Buzzard-Diamond-Jarvis conjecture on when a given two dimensional, mod $p$ global Galois representation is modular of weight $\sigma_{\underline{n}, \underline{m}}$.

2.3.1. Suppose now that $K / \mathbb{Q}_{p}$ is unramified. We will give the explicit values of $\mu_{\underline{n}, \underline{m}}\left(V_{\mathbb{F}}\right)$ when $V_{\mathbb{F}}$ is absolutely irreducible.

Let $K^{\prime} / K$ be the unramified extension of degree 2 , so that $I_{K}=I_{K^{\prime}}=I_{\mathbb{Q}_{p}}$. Let $k^{\prime}$ denote the residue field of $K^{\prime}$. Let $n=\left[K: \mathbb{Q}_{p}\right]$ and $\omega_{2 n}: I_{\mathbb{Q}_{p}} \rightarrow k^{\prime \times}$ the fundamental character of level $2 n$ and $\omega_{n}=\omega_{2 n}^{p^{n}+1}$ the fundamental character of level $n$. We will assume that $E$ contains all embeddings of $K^{\prime}$ into $\overline{\mathbb{Q}}_{p}$.

Let $J$ be a subset of the embeddings $k^{\prime} \hookrightarrow \mathbb{F}$ which bijects onto the set of all embeddings $k \hookrightarrow \mathbb{F}$. We set

$$
\omega_{J}=\prod_{\bar{\iota} \in J} \iota \circ\left(\omega_{2 n}^{\underline{n}_{\iota}+1} \cdot \omega_{n}^{\frac{m}{n}}\right),
$$

where for $\iota \in J$ we again denote by $\iota$ the restriction of $\iota$ to $k$. Thus $\omega_{J}$ is a character $I_{K} \rightarrow \mathbb{F}^{\times}$. Similarly, if $J^{\prime}$ denotes the compliment of $J$ in the set of embeddings $\bar{\iota}: k^{\prime} \hookrightarrow \mathbb{F}$, we have the character $\omega_{J^{\prime}}$.

Conjecture 2.3.2. Suppose $V_{\mathbb{F}}$ is absolutely irreducible. Then Conjecture 2.2.3 holds with $\mu_{\underline{n}, \underline{m}}\left(V_{\mathbb{F}}\right)=0$ unless there exists $J$ as above such that

$$
\left.V_{\mathbb{F}}\right|_{I_{K}} \sim\left(\begin{array}{cc}
\omega_{J} & 0 \\
0 & \omega_{J^{\prime}}
\end{array}\right)
$$

in which case $\mu_{\underline{\underline{n}}, \underline{m}}\left(V_{\mathbb{F}}\right)=1$.

\section{Theorems}

3.1. Statements. We will review some cases when Conjecture 2.2 .3 is known as well as sketching some of the arguments. We assume from now on that $p>2$. 
Most of the conjecture is known when $K=\mathbb{Q}_{p}$. In this case each of $\underline{n}, \underline{m}$ consist of a single integer which we denote by $n$ and $m$ respectively, and we write $\mu_{n, m}\left(V_{\mathbb{F}}\right)$ for $\mu_{\underline{n}, \underline{m}}\left(V_{\mathbb{F}}\right)$. The explicit value of $\mu_{n, m}\left(V_{\mathbb{F}}\right)$ is known in all cases, except when $n=p-2$ and $V_{\mathbb{F}}$ is scalar. One has the following result [Ki 5], which, in particular includes (most of) the original conjecture stated by Breuil-Mézard (here $\omega$ denotes the mod $p$ cyclotomic character).

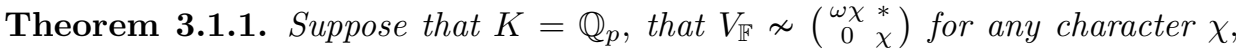
and that if $V_{\mathbb{F}}$ has scalar semi-simplification then it is scalar.

Then Conjecture 2.2.3 holds for any regular $\mathbf{v}$ and any $\tau$.

3.1.2. The proof uses the $p$-adic local Langlands correspondence for $\mathrm{GL}_{2}\left(\mathbb{Q}_{p}\right)$ to prove that the left hand side in the equalities in Conjecture 2.2.3 is bounded above by the right hand side. To each two dimensional $E$-representation $V_{E}$ of $G_{\mathbb{Q}_{p}}$, this correspondence attaches a certain representation of $\mathrm{GL}_{2}\left(\mathbb{Q}_{p}\right)$ on a $p$-adic Banach space $\Pi(V)$. A key ingredient in the proof is the fact that the $p$-adic local Langlands correspondence is compatible with the usual local Langlands correspondence, in the sense that, if $V_{E}$ is potentially semi-stable with $p$-adic Hodge type $\mathbf{v}$ and Galois type $\tau$, then the locally algebraic vectors in $\Pi(V)$ contain a copy of the $\mathrm{GL}_{2}\left(\mathbb{Z}_{p}\right)$ representation $\sigma(\mathbf{v}, \tau)$. This was proved by Colmez and Berger-Breuil [Co 2], [BB] when $\tau$ arises from an abelian representation of the Weil group, and by Colmez $[\mathrm{Co}]$ in general, using Emerton's work on the local-global compatibility of the $p$-adic Langlands correspondence [Em].

The opposite inequality is proved by a Taylor-Wiles style patching argument. Indeed, this patching argument shows that Conjecture 2.2.3 is very closely related to the conjecture of Fontaine-Mazur on the modularity of geometric Galois representations. One can attempt to run this argument in reverse and deduce Conjecture 2.2.3 from a modularity lifting theorem. For potentially Barsotti-Tate representations such a theorem was proved in $[\mathrm{Ki} 1]$ and generalized by Gee [Ge 1]. Using it one can show that for any $K / \mathbb{Q}_{p}$ we have

Theorem 3.1.3. Denote by $\mathbf{v}_{0}$ the cocharacter corresponding to $k_{\iota}-1=w_{\iota}=0$ for all $\iota$. If $V_{\mathbb{F}}$ is absolutely irreducible, then there exist non-negative integers $\mu_{\underline{n}, \underline{m}}\left(V_{\mathbb{F}}\right)$ such that for any $\tau$,

$$
e\left(R_{\mathrm{cr}}^{\square, \mathbf{v}_{0}, \tau, \psi} / \pi\right)=\sum_{\underline{n}, \underline{m}} a(\underline{n}, \underline{m}) \mu_{\underline{n}, \underline{m}}\left(V_{\mathbb{F}}\right),
$$

where

$$
\left.\left(L_{\mathbf{v}_{0}, \tau}^{\mathrm{cr}} \otimes_{\mathcal{O}} \mathbb{F}\right)^{\mathrm{ss}} \stackrel{\sim}{\longrightarrow} \oplus_{\underline{n}, \underline{m}} \sigma_{\underline{n}, \underline{\underline{n}}}^{a(\underline{\underline{n}}}\right) .
$$

3.1.4. Now return to the case where $K / \mathbb{Q}_{p}$ is unramified. We assume that $V_{\mathbb{F}}$ is absolutely irreducible, and we now take $\mu_{\underline{n}, \underline{m}}\left(V_{\mathbb{F}}\right)$ to be defined as in Conjecture 2.3 .2 , so that $\mu_{\underline{n}, \underline{m}}\left(V_{\mathbb{F}}\right)$ is non-zero if and only if there exists $J$ such that $\left.V_{\mathbb{F}}\right|_{I_{K}} \sim$ $\left(\begin{array}{cc}\omega_{J} & 0 \\ 0 & \omega_{J^{\prime}}\end{array}\right)$ in which case $\mu_{\underline{n}, \underline{m}}\left(V_{\mathbb{F}}\right)=1$.

We will say that $\mathbf{v}$ is paritious if the integers $k_{\iota}+2 w_{\iota}$ are independent of $\iota$. We will say that $V_{\mathbb{F}}$ is regular, if there exists $(\underline{n}, \underline{m})$ with $\mu_{\underline{n}, \underline{m}}\left(V_{\mathbb{F}}\right) \neq 0$ and $2 \leq n_{\iota} \leq p-4$ for all $\iota$. 
Theorem 3.1.5. Suppose that $K / \mathbb{Q}_{p}$ is unramified, that $\mathbf{v}$ is paritious and that $V_{\mathbb{F}}$ is absolutely irreducible and regular. Then

$$
e\left(R^{\mathbf{v}, \tau, \psi} / \pi\right) \geq \sum_{\underline{n}, \underline{m}} a(\underline{n}, \underline{m}) \mu_{\underline{n}, \underline{m}}\left(V_{\mathbb{F}}\right),
$$

where

$$
\left.\left(L_{\mathbf{v}, \tau} \otimes_{\mathcal{O}} \mathbb{F}\right)^{\mathrm{ss}} \stackrel{\sim}{\longrightarrow} \oplus_{\underline{n}, \underline{m}} \sigma_{\underline{n}, \underline{\underline{n}}, \underline{\underline{m}}}^{a(}\right),
$$

and similarly for $e\left(R_{\mathrm{cr}}^{\mathbf{v}, \tau, \psi} / \pi\right)$.

3.2. A sketch of the proofs. We now give a sketch of some of the methods which are used to prove Theorems 3.1.3 and 3.1.5. These involve relating the Hilbert-Samuel multiplicities in the conjectures to those of certain spaces of automorphic forms.

It ought to be possible to extend these methods to prove Conjecture 2.2.3 for $e\left(R_{\mathrm{cr}}^{\square, \mathbf{v}, \tau, \psi} / \pi\right)$ with an explicit collection of integers $\mu_{\underline{n}, \underline{m}}\left(V_{\mathbb{F}}\right)$, when $\mathbf{v}=\mathbf{v}_{0}$ and $K / \mathbb{Q}_{p}$ is unramified. This is work in progress with Toby Gee.

3.2.1. Let $F$ be a totally real number field and $D$ a totally definite quaternion algebra over $F$, which is unramified at all primes $v \mid p$ of $F$. Denote by $\mathbb{A}_{F}^{f} \subset \mathbb{A}_{F}$ the finite adeles. For each finite place $v$ of $F$ we will denote by $\pi_{v} \in F_{v}$ a uniformizer. Fix a maximal order $\mathcal{O}_{D} \subset D$, and an isomorphism $\left(\mathcal{O}_{D}\right)_{v} \stackrel{\sim}{\longrightarrow} M_{2}\left(\mathcal{O}_{F_{v}}\right)$ for each finite place where $D$ is unramified. Let $U=\prod_{v} U_{v} \subset\left(D \otimes_{F} \mathbb{A}_{F}^{f}\right)^{\times}$be a compact open subgroup contained in $\prod_{v}\left(\mathcal{O}_{D}\right)_{v}^{\times}$. We assume that $U_{v}=\mathrm{GL}_{2}\left(\mathcal{O}_{F_{v}}\right)$ for $v \mid p$.

For each $v \mid p$, we fix a continuous representation $\sigma_{v}: U_{v} \rightarrow \operatorname{Aut}\left(W_{\sigma_{v}}\right)$ on a finite $\mathcal{O}$-module. Write $W_{\sigma}=\otimes_{v \mid p, \mathcal{O}} W_{\sigma_{v}}$ and denote by $\sigma: \prod_{v \mid p} U_{v} \rightarrow \operatorname{Aut}\left(W_{\sigma}\right)$ the corresponding representation. We regard $\sigma$ as being a representation of $U$ by letting $U_{v}$ act trivially if $v \nmid p$. Finally, assume there exists a continuous character $\psi:\left(\mathbb{A}_{F}^{f}\right)^{\times} / F^{\times} \rightarrow \mathcal{O}^{\times}$such that $\sigma$ on $U \cap\left(\mathbb{A}_{F}^{f}\right)^{\times}$is given by multiplication by $\psi$. Fix such a $\psi$, and extend the action of $U$ on $W_{\sigma}$ to $U\left(\mathbb{A}_{F}^{f}\right)^{\times}$, by letting $\left(\mathbb{A}_{F}^{f}\right)^{\times}$act via $\psi$.

Let $S_{\sigma, \psi}(U)$ denote the set of continuous functions

$$
f: D^{\times} \backslash\left(D \otimes_{F} \mathbb{A}_{F}^{f}\right)^{\times} \rightarrow W_{\sigma}
$$

such that for $g \in\left(D \otimes_{F} \mathbb{A}_{F}^{f}\right)^{\times}$we have $f(g u)=\sigma(u)^{-1} f(g)$ for $u \in U$, and $f(g z)=\psi^{-1}(z) f(g)$ for $z \in\left(\mathbb{A}_{F}^{f}\right)^{\times}$.

We consider the left action of $\left(D \otimes_{F} \mathbb{A}_{F}^{f}\right)^{\times}$on $W_{\sigma}$-valued functions on $\left(D \otimes_{F}\right.$ $\left.\mathbb{A}_{F}^{f}\right)^{\times}$given by the formula $(g f)(z)=f(z g)$. Then for any finite prime $v$, the double cosets of $U_{v}$ in $\left(D \otimes_{F} \mathbb{A}_{F}^{f}\right)^{\times}$act naturally on $S_{\sigma, \psi}(U)$. Denote by $\mathbb{T}_{\sigma, \psi}(U)$ the $\mathcal{O}$ algebra generated by the endomorphisms $S_{v}$ and $T_{v}$ of $S_{\sigma, \psi}(U)$ corresponding to $U_{v}\left(\begin{array}{cc}\pi_{v} & 0 \\ 0 & \pi_{v}\end{array}\right) U_{v}$ and $U_{v}\left(\begin{array}{cc}\pi_{v} & 0 \\ 0 & 1\end{array}\right) U_{v}$ respectively, where $v \nmid p$ runs over primes at which $D$ is unramified. If $U_{v}$ is maximal compact in $\left(D \otimes_{F} F_{v}\right)^{\times}$, then these operators do not depend on the choice of $\pi_{v}$. 
3.2.2. Now fix an algebraic closure $\bar{F}$ of $F$ and let $S$ be a finite set of primes of $F$, containing the infinite primes, the primes dividing $p$, the primes where $D$ is ramified, and the primes where $U_{v}$ is not maximal compact in $\left(D \otimes_{F} F_{v}\right)^{\times}$. Let $F_{S} \subset \bar{F}$ be the maximal extension of $F$ unramified outside $S$, and set $G_{F, S}=$ $\operatorname{Gal}\left(F_{S} / F\right)$.

Let $\mathfrak{m} \subset \mathbb{T}_{\sigma, \psi}(U)$ be a maximal ideal. Such an ideal is called Eisenstein if $T_{v}-2 \in \mathfrak{m}$ for all but finitely many primes $v \notin S$ which split completely in some fixed abelian extension of $F$. After possibly replacing $\mathcal{O}$ by an extension we may assume that $\mathfrak{m}$ has residue field $\mathbb{F}$. If $\mathfrak{m}$ is a non-Eisenstein ideal, then the work of Carayol [Ca] and Taylor [Ta], together with the Jacquet-Langlands correspondence, implies that there exists a unique representation

$$
\rho_{\mathfrak{m}}: G_{F, S} \rightarrow \mathrm{GL}_{2}\left(\mathbb{T}_{\sigma, \psi}(U)_{\mathfrak{m}}\right)
$$

such that if $v \notin S$ is a prime of $F$, and Frob $_{v}$ denotes an arithmetic Frobenius at $v$ then $\rho_{\mathfrak{m}}\left(\right.$ Frob $\left._{v}\right)$ has trace $T_{v}$. We denote by $\bar{\rho}_{\mathfrak{m}}$ the reduction of $\rho_{\mathfrak{m}}$ modulo $\mathfrak{m}$. As $\mathfrak{m}$ is non-Eisenstein $\bar{\rho}_{\mathfrak{m}}$ is absolutely irreducible.

3.2.3. Now suppose we are given $\mathbf{v}$ and $\tau$ as in section 2.1 .2 with $\mathbf{v}$ paritious and an absolutely irreducible representation $V_{\mathbb{F}}$ of $G_{K}$. Then we choose $F$ such that there is a unique prime $\mathfrak{p} \mid p$ of $F$ and $F_{\mathfrak{p}} \stackrel{\sim}{\longrightarrow} K$. Fix an embedding $\bar{F} \hookrightarrow \bar{K}$, extending this isomorphism. We choose the character $\psi:\left(\mathbb{A}_{F}^{f}\right)^{\times} / F^{\times} \rightarrow \mathcal{O}^{\times}$so that $\left.\psi\right|_{I_{K}}=\left.\chi_{\mathbf{v}}\right|_{I_{K}} \operatorname{det} \tau$, and we apply the above constructions with $\sigma$ a $\operatorname{GL}_{2}\left(\mathcal{O}_{K}\right)$ stable $\mathcal{O}$-lattice $L_{\mathbf{v}, \tau}^{\mathrm{cr}}$ in $\sigma_{\mathrm{cr}}(\mathbf{v}, \tau)$.

Using CM forms, one can find $\mathfrak{m}$ such that $\left.\bar{\rho}_{\mathfrak{m}}\right|_{G_{K}} \sim V_{\mathbb{F}}$, and we again denote by $V_{\mathbb{F}}$ the underlying $\mathbb{F}$-vector space of $\bar{\rho}_{\mathfrak{m}}$.

Let $R_{F, S}$ and $R_{\mathfrak{p}}$ denote the the universal deformation rings of $V_{\mathbb{F}}$ and $\left.V_{\mathbb{F}}\right|_{G_{K}}$ respectively. We denote by $R_{F, S}^{\psi}$ the quotient of $R_{F, S}$ which parameterizes deformations of determinant $\psi \chi_{\mathrm{cyc}}$, where $\chi_{\text {cyc }}$ now denotes the $p$-adic cyclotomic character on $G_{F, S}$. Set

$$
R_{F, S}^{\mathbf{v}, \tau, \psi}=R_{V_{\mathbb{F}}, \mathrm{cr}}^{\mathbf{v}, \tau, \psi} \otimes_{R_{\mathfrak{p}}} R_{F, S}^{\psi}
$$

The map

$$
R_{F, S} \rightarrow \mathbb{T}_{\sigma, \psi}(U)_{\mathfrak{m}}
$$

induced by $\rho_{\mathfrak{m}}$, factors through $R_{F, S}^{\mathbf{v}, \tau, \psi}$. (See for example [Ki $\left.4, \S 4\right]$.)

Under some technical restrictions on the choice of $F, D$ and $U$, which can always be arranged for a given representation $V_{\mathbb{F}}$ of $G_{K}$, a Taylor-Wiles patching argument, as modified by Diamond [Di] and Fujiwara, and in [Ki 1, §3], [Ki 5, §2], shows that there exist an $\mathcal{O}$-algebra $R_{\infty}$, maps of $\mathcal{O}$-algebras

$$
\mathcal{O} \llbracket y_{1}, \ldots, y_{h} \rrbracket \rightarrow R_{V_{\mathbb{F}}, \mathrm{cr}}^{\mathbf{v}, \tau, \psi} \llbracket x_{1}, \ldots, x_{h-d} \rrbracket \rightarrow R_{\infty},
$$

and an $R_{\infty}$-module $M_{\infty}$ satisfying the following properties:

(1) $h \geq d=\operatorname{dim} R_{V_{\mathbb{F}}, \mathrm{cr}}^{\mathbf{v}, \tau} / \pi=\left[K: \mathbb{Q}_{p}\right]$.

(2) There is an isomorphism of $R_{V_{\mathbb{F}}, \mathrm{cr}}^{\mathbf{v}, \tau, \psi}$ algebras $R_{\infty} /\left(y_{1}, \ldots, y_{h}\right) \stackrel{\sim}{\longrightarrow} R_{F, S}^{\mathbf{v}, \tau, \psi}$. 
(3) $M_{\infty}$ is a finite free $\mathcal{O} \llbracket y_{1}, \ldots, y_{h} \rrbracket$-module and has rank at most 1 on any irreducible component on $\operatorname{Spec} R_{V_{\mathbb{F}}, \mathrm{cr}}^{\mathbf{v}, \tau} \llbracket x_{1}, \ldots, x_{h-d} \rrbracket$.

(4) There is an isomorphism of $R_{F, S}^{\mathbf{v}, \tau, \psi}$-modules

$$
M_{\infty} /\left(y_{1}, \ldots, y_{h}\right) M_{\infty} \stackrel{\sim}{\longrightarrow} S_{\sigma, \psi}(U)_{\mathfrak{m}}
$$

Now let

$$
\{0\}=M^{0} \subset M^{1} \subset \cdots \subset M^{s}=L_{\mathbf{v}, \tau}^{\mathrm{cr}} / \pi
$$

be a filtration such that $M^{i+1} / M^{i}$ is an irreducible representation of $\mathrm{GL}_{2}(k)$. Then we can enhance the above construction (see $[\mathrm{Ki} 5,2.2 .9]$ ) in such a way that there exists a filtration

$$
\{0\}=M_{\infty}^{0} \subset M_{\infty}^{1} \subset \cdots \subset M_{\infty}^{s}=M_{\infty} / \pi M_{\infty}
$$

by $R_{\infty}$-modules such that

(5) $M_{\infty}^{i} / M_{\infty}^{i-1}$ is a finite free $\mathbb{F} \llbracket y_{1}, \ldots, y_{h} \rrbracket$-module.

(6) If $M^{i} / M^{i-1} \stackrel{\sim}{\longrightarrow} \sigma_{\underline{n}, \underline{m}}$ then the isomorphism in (4) above induces an isomorphism

$$
M_{\infty}^{i} / M_{\infty}^{i-1} \otimes_{R_{\infty}} R_{\infty} /\left(y_{1}, \ldots, y_{h}\right) \stackrel{\sim}{\longrightarrow} S_{\sigma_{\underline{n}, \underline{m}}, \psi}(U)_{\mathfrak{m}}
$$

Moreover this construction can be made so that, as an $R_{\mathfrak{p}} \llbracket x_{1}, \ldots, x_{h-d} \rrbracket$-module, $M_{\infty}^{i} / M_{\infty}^{i-1}$ depends only on $\sigma_{\underline{n}, \underline{m}}$ and $\mathfrak{m}$, and not on the choice of $\mathbf{v}$ and $\tau$. More precisely this module is made by an analogous patching argument but with $\sigma_{\underline{n}, \underline{m}}$ in place of $L_{\mathbf{v}, \tau}^{\mathrm{cr}}$. We denote this module by $M_{\infty}^{\underline{n}, \underline{m}}$.

Set $R_{\infty}^{\prime}=R_{V_{\mathbb{F}}, \mathrm{cr}}^{\mathbf{v}, \tau, \psi} \llbracket x_{1}, \ldots, x_{h-d} \rrbracket$, and let $a(\underline{n}, \underline{m})$ be the multiplicity with which $\sigma_{\underline{n}, \underline{m}}$ appears as a Jordan-Hölder factor in $L_{\mathbf{v}, \tau}^{\mathrm{cr}} / \pi$. Using (3) and (5) and standard facts about Hilbert-Samuel multiplicities one obtains

$$
e\left(R_{V_{\mathbb{F}}, \mathrm{cr}}^{\mathbf{v}, \tau, \psi} / \pi\right)=e\left(R_{\infty}^{\prime} / \pi R_{\infty}^{\prime}\right) \geq e_{R_{\infty}^{\prime} / \pi}\left(M_{\infty} / \pi M_{\infty}\right)=\sum_{\underline{n}, \underline{m}} a(\underline{n}, \underline{m}) e_{R_{\infty}^{\prime} / \pi}\left(M_{\infty}^{\underline{n}, \underline{m}}\right) .
$$

with equality if and only if Spec $R_{\infty}^{\prime}[1 / p]$ is contained in the support of the $R_{\infty}^{\prime}$ module $M_{\infty}$ (cf. [Ki 5, Lem. 2.2.11]). Note that the freeness condition in (3) implies that this support is a union of irreducible components of Spec $R_{\infty}^{\prime}[1 / p]$ as the dimensions of $\mathcal{O} \llbracket y_{1}, \ldots, y_{h} \rrbracket$ and $R_{\infty}^{\prime}$ coincide by (1). This also implies that $e_{R_{\infty}^{\prime} / \pi}\left(M_{\infty}^{\underline{n}, \underline{m}}\right)$ depends only on the image of $R_{\mathfrak{p}} \llbracket x_{1}, \ldots, x_{h-d} \rrbracket$ in End $M_{\infty}^{\underline{n}, \underline{m}}$ and not on $R_{\infty}^{\prime}$, and is therefore independent of $\mathbf{v}$ and $\tau$.

3.2.6. Proof of Theorem 3.1.5. In this case $K / \mathbb{Q}_{p}$ is unramified and $V_{\mathbb{F}}$ is assumed regular. We have to show that

$$
e_{R_{\infty}^{\prime} / \pi}\left(M_{\infty}^{\underline{n}, \underline{m}}\right) \geq \mu_{\underline{n}, \underline{m}}\left(V_{\mathbb{F}}\right)
$$


By definition, the term on the right is 0 or 1 , and in the former case there is nothing to prove. Suppose $\mu_{n, m}\left(V_{\mathbb{F}}\right)=1$. As above, the condition (5) implies that the support of $M_{\infty}^{\underline{n}, \underline{m}}$ has dimension equal to $\operatorname{dim} R_{\infty}^{\prime} / \pi$. Hence it suffices to show that $M_{\infty}^{\underline{n}, \underline{m}} \neq\{0\}$. By (6) it suffices to show that $S_{\sigma_{\underline{n}, \underline{m}}, \psi}(U)_{\mathfrak{m}} \neq\{0\}$. This follows from Gee's proof [Ge 2] of the Buzzard-Diamond-Jarvis conjecture for regular weights. Namely our condition on the regularity of $V_{\mathbb{F}}$ implies that any $\sigma_{\underline{n}, \underline{m}}$ such that $\mu_{\underline{n}, \underline{m}}\left(V_{\mathbb{F}}\right) \neq 0$ is regular in the sense of [Ge 2].

This completes the proof of Theorem 3.1.5 for $R_{V_{\mathbb{F}}, \mathrm{vr}}^{\mathbf{v}, \tau}$ and the proof for $R_{V_{\mathbb{F}}}^{\mathbf{v}, \tau, \psi}$ is identical, replacing $L_{\mathbf{v}, \tau}^{\mathrm{cr}}$ by a $\mathrm{GL}_{2}\left(\mathcal{O}_{K}\right)$-invariant lattice in $\sigma(\mathbf{v}, \tau)$.

3.2.8. Proof of Theorem 3.1.3: Let $\mathbf{v}=\mathbf{v}_{0}$, and set $\mu_{n, m}\left(V_{\mathbb{F}}\right)=e_{R_{\infty}^{\prime}}\left(M_{\infty}^{\underline{n}, \underline{m}}\right)$. To prove the theorem we have to show that the inequality in (3.2.5) is an equality. It is enough to show that $M_{\infty}$ is a faithful $R_{\infty}^{\prime}$-module.

The following lemma will be useful.

Lemma 3.2.9. The following are equivalent

(1) The support of $S_{\sigma, \psi}(U)_{\mathfrak{m}}$ contains $\operatorname{Spec} R_{F, S}^{\mathbf{v}, \tau, \psi}[1 / p]$ and $R_{F, S}^{\mathbf{v}, \tau, \psi}$ is a finite $\mathcal{O}$ algebra.

(2) $M_{\infty}$ is a faithful $R_{\infty}^{\prime}$-module.

Proof. (2) $\Longrightarrow$ (1): If $M_{\infty}$ is a faithful $R_{\infty}^{\prime}$-module then $R_{\infty}^{\prime}=R_{\infty}$ and both are finite over $\mathcal{O} \llbracket y_{1}, \ldots, y_{h} \rrbracket$. Then (1) follows from conditions (2) and (4) in (3.2.3).

$(1) \Longrightarrow(2)$ : One can use an argument of Khare-Wintenberger [KW 2, Cor. 4.7] to show that the second condition in (1) implies that the image of $\operatorname{Spec} R_{F, S}^{\mathbf{v}, \tau, \psi}[1 / p]$ in Spec $R_{V_{\mathbb{F}}, \mathrm{vr}}^{\mathbf{v}, \tau, \psi}[1 / p]$ meets every irreducible component of the latter scheme. Hence the first condition implies that the support of $S_{\sigma, \psi}(U)_{\mathfrak{m}}$ meets every irreducible component of $R_{\infty}^{\prime}$. Since the support of $M_{\infty}$ is a union of irreducible components of Spec $R_{\infty}^{\prime}[1 / p]$, it must contain all of Spec $R_{\infty}^{\prime}[1 / p]$ by condition (4) in (3.2.3). Finally as $R_{\infty}^{\prime}$ is flat over $\mathcal{O}$ with formally smooth (so in particular reduced) generic fibre, this implies that $M_{\infty}$ is a faithful $R_{\infty}^{\prime}$-module.

3.2.10. We return to the proof of Theorem 3.1.3. Since $\mathbf{v}=\mathbf{v}_{0}$ the main result of [Ki 1] and [Ge 1] shows that the support of $S_{\sigma, \psi}(U)_{\mathfrak{m}}$ contains $\operatorname{Spec} R_{F, S}^{\mathbf{v}, \tau, \psi}[1 / p]$.

Moreover the proof in loc. cit (cf. also [Ki 3, §1]) together with an argument of Khare-Wintenberger [KW 1, Prop. 3.8] shows that that $R_{F, S}^{\mathbf{v}, \tau, \psi}$ is a finite $\mathcal{O}$-algebra. More precisely, the argument in $[\mathrm{Ki} 1, \S 3.4]$ carries out a patching argument analogous to the one sketched here, but over a finite, solvable, totally real extension $F^{\prime} / F$. In that situation the analogue of the $\operatorname{ring} R_{V_{\mathrm{F}}, \mathrm{cr}}^{\mathbf{v}_{0}, \tau}$ turns out to be a domain. This implies that the analogue of the condition (2) in Lemma 3.2.9 is automatically satisfied, and hence so is the condition (1). This is enough to imply the finiteness of $R_{F, S}^{\mathbf{v}, \tau, \psi}$ itself. 


\section{References}

[BB] L. Berger, C. Breuil, Sur quelques représentations potentiellement cristallines de $\mathrm{GL}_{2}\left(\mathbb{Q}_{p}\right)$, Astérisque, 330, 155-211, 2010.

[BCDT] C. Breuil, B. Conrad, F. Diamond, R. Taylor, On the modularity of elliptic curves over $\mathbb{Q}$ : wild 3-adic exercises, J. Amer. Math. Soc. 14, 843-939, 2001.

[BDJ] K. Buzzard, F. Diamond, F. Jarvis, On Serre's conjecture for mod l Galois representations over totally real fields, Duke Math. J, to appear, 2010.

[Be] L. Berger, Limites des représentations cristallines, Compositio Math. 140, 1473-1498, 2004.

[BL] L. Barthel, R. Livne, Irreducible modular representations of $\mathrm{GL}_{2}$ of a local field, Duke Math. J, 75, 261-292, 1994.

[BM] C. Breuil, A. Mézard, Multiplicités modulaires et représentations de $\mathrm{GL}_{2}\left(\mathbb{Z}_{p}\right)$ et de $\operatorname{Gal}\left(\overline{\mathbb{Q}}_{p} / \mathbb{Q}_{p}\right)$ en $l=p$, Duke Math. J. 115, 205-310, 2002, with an appendix by G. Henniart.

[Br 1] C. Breuil, Introduction Générale, Astérisque 319, 1-12, 2008.

[Br 2] C. Breuil, Représentations semi-stables et modules fortement divisibles, Invent. Math. 136, 89-122, 1999.

[Ca] H. Carayol, Sur les représentations l-adiques associes aux formes modulaires de Hilbert, Ann. Sci. École Norm. Sup, 19, 409-468, 1986.

$[\mathrm{Co}] \quad$ P. Colmez, Représentations de $\mathrm{GL}_{2}\left(\mathbb{Q}_{p}\right)$ et $(\varphi, \Gamma)$-modules, Astérisque, 330, 283-511, 2010.

[Co2] P. Colmez La série principal unitaire pour $\mathrm{GL}_{2}\left(\mathbb{Q}_{p}\right)$. Astérisque, 330, 213-262, 2010 .

[Di] F. Diamond, The Taylor-Wiles construction and multiplicity one, Invent. Math. 128, 379-391, 1997.

[Em] M. Emerton Local-global compatibility in the p-adic Langlands program for $\mathrm{GL}_{2 / \mathbb{Q}}$, In preparation.

[FL] J-M. Fontaine, G. Laffaille, Construction de répresentations p-adiques, Ann. Scient. de l'E.N.S. 15, 547-608, 1982.

[FM] J.M. Fontaine, B. Mazur, Geometric Galois Representations, Elliptic curves, modular forms, and Fermat's last theorem (Hong Kong 1993), 41-78, Internat. Press, Cambridge MA, 1995.

[Fo 1] J.M. Fontaine, Représentations p-adiques semi-stables, Périodes p-adiques, Astérisque 223, 11-184, Société Mathématique de France, 1994.

[Fo 2] J.M. Fontaine, Deforming semi-stable Galois representations, Proc. Natl. Acad. Sci. USA, 94 11138-11141, 1997.

[Ge 1] T. Gee, A modularity lifting theorem for weight two modular forms, Math. Res. Lett. 13, 805-811, 2006.

[Ge 2] T. Gee, On the weights of mod $p$ Hilbert modular forms, preprint, 2006.

[Ki 1] M. Kisin, Moduli of finite flat group schemes and modularity, Ann. of Math. 170(3), 1085-1180, 2009. 
[Ki 2] M. Kisin, Crystalline representations and F-crystals, Algebraic geometry and number theory. In honor of Vladimir Drinfeld's $50^{\text {th }}$ birthday, Prog. Math. 253, 459-496, Birkhäuser, 2006.

[Ki 3] M. Kisin, Modularity of some geometric Galois representations, L-functions and Galois representations (Durham 2004), LMS 320, 438-470, Cambridge Univ. Press, 2007.

[Ki 4] M. Kisin, Potentially semi-stable deformation rings, J. AMS, 21, 513-546, 2008.

[Ki 5] M. Kisin, The Fontaine-Mazur conjecture for $\mathrm{GL}_{2}$, J. AMS, 22, 641-690, 2009.

$[\mathrm{KW} 1] \quad$ C. Khare, J.P. Wintenberger, On Serre's conjecture for 2-dimensional mod $p$ representations of $\operatorname{Gal}(\overline{\mathbb{Q}} / \mathbb{Q})$, Ann. of Math, 169, 229-253, 2009.

[KW 2] C. Khare, J.P. Wintenberger, Serre's modularity conjecture (II), Invent. Math, 178(3), 505-586, 2009.

[Li] T. Liu, Torsion p-adic Galois representations and a conjecture of Fontaine, Ann. Sci. École Norm. Sup, 40(4), 633-674, 2007.

[Ma] B. Mazur, Deforming Galois representations, Galois groups over $\mathbb{Q}$ (Berkeley, CA, 1987), Math. Sci. Res. Inst. Publ. 16, 395-437, Springer, New York-Berlin, 1989.

[Pa] V. Paskunas Unicity of types for supercuspidal representations of $\mathrm{GL}_{N}$, Proc. LMS (3) 91, 623-654, 2005.

[Ra] R. Ramakrishna, On a variation of Mazur's deformation functor, Compositio Math. 87, 269-286, 1993.

[Ta] R. Taylor, On Galois representations associated to Hilbert modular forms, Invent. Math. 98, 265-280, 1989.

[Wi] A. Wiles, Modular elliptic curves and Fermat's last theorem, Ann. of Math. 141(3), 443-551, 1995.

[TW] R. Taylor, A. Wiles, Ring theoretic properties of certain Hecke algebras, Ann. of Math. 141(3), 553-572, 1995.

Department of Mathematics, Harvard,

1 Oxford st, Cambridge MA 02139, USA.

E-mail: kisin@math.harvard.edu 